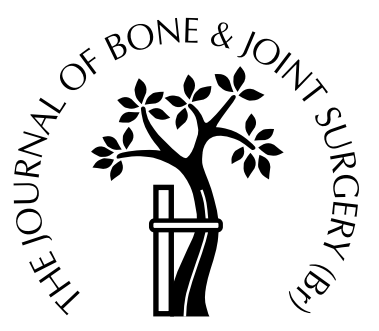

\title{
New radiographic projections for avulsion fractures of the lateral malleolus
}

\author{
Naoki Haraguchi, Fumio Kato, Hiromichi Hayashi \\ From the West Tokyo Metropolitan Police Hospital, Tokyo, Japan
}

W e report two new radiographic projections for evaluating avulsion fractures at the lateral malleolus. We used seven freshly amputated legs with simulated avulsion fractures and radiopaque markers to assess their value.

The projections allow accurate assessment of the displacement of fragments without superimposition, and also show whether they affect the anterior talofibular or the calcaneofibular ligament or both.

J Bone Joint Surg [Br] 1998;80-B:684-8.

Received 15 November 1997; Accepted 23 February 1998

Avulsion fractures of the lateral malleolus do not always unite in a cast. There are usually no symptoms, but sometimes there is pain and instability, ${ }^{1-5}$ especially in very active patients. Both the anterior talofibular (ATFL) and the calcaneofibular (CFL) ligaments arise from the anteroinferior aspect of the lateral malleolus, and therefore avulsion fragments are superimposed on the lateral malleolus on standard radiographs, and are difficult to identify accurately.

We aimed to develop a better technique for evaluating these injuries.

\section{Materials and Methods}

We used seven legs amputated for peripheral vascular disease or diabetes mellitus. Soft tissues were removed to expose the insertions of the ATFL and the CFL. We marked them with soft wires glued around both bony attachments and closely tied around the ligaments. We identified the midpoints of the medial and lateral malleoli in the sagittal

\footnotetext{
N. Haraguchi, MD, Orthopaedic Surgeon

F. Kato, MD, Chief Orthopaedic Surgeon

Department of Orthopaedic Surgery, Tokyo Metropolitan Police Hospital,

2-10-41 Fujimi, Chiyoda-ku, Tokyo 102, Japan.

H. Hayashi, MD, Chief Orthopaedic Surgeon

Department of Orthopaedic Surgery, West Tokyo Metropolitan Police

Hospital, 4-8-1 Nishimoto-cho, Kokubunji-shi, Tokyo 185, Japan.

Correspondence should be sent to Dr N. Haraguchi.

(C)1998 British Editorial Society of Bone and Joint Surgery

0301-620X/98/48636\$2.00
}

plane using a calliper, then passed a Kirschner wire through these points parallel to the tibial joint surface to define the transmalleolar axis.

Radiographs of the prepared feet were taken with the central beam directed vertical to the cassette at the same focal distance; all positioning was done by one author $(\mathrm{NH})$ who measured the relevant angles with a goniometer.

Projection 1. The sole of the specimen was placed firmly on the cassette, at first with the foot in neutral position, at $90^{\circ}$ to the distal leg. The medial side of the foot was then elevated progressively so that the Kirschner wire was at $0^{\circ}$, $15^{\circ}, 30^{\circ}, 45^{\circ}$ and then $60^{\circ}$ from the horizontal; the lateral border of the foot was kept in contact with the cassette. At each horizontal angle, films were taken with plantar flexion of the ankle to $15^{\circ}, 30^{\circ}$ and $45^{\circ}$.

Projection 2. Specimens were placed with the posterior heel resting on the cassette and the ankle in a neutral position. The leg and foot were then rotated medially and films taken with the Kirschner wire at angles of $0^{\circ}, 15^{\circ}$, $30^{\circ}, 45^{\circ}, 60^{\circ}$ and $75^{\circ}$ to the horizontal. The central beam was directed towards the lateral malleolus.

Simulation of avulsion fractures. Preliminary results showed that radiographs taken at $15^{\circ}$ external rotation with $45^{\circ}$ of plantar flexion in projection 1 , and at $45^{\circ}$ internal rotation in projection 2 were the most useful. Only radiographs taken at these angles were used for the second phase of our study. In four specimens, avulsion fractures of the ATFL were simulated by osteotomy of the lateral malleolus, and in three specimens, avulsion fractures of the CFL were simulated. The radiographs were then repeated.

\section{Results}

ATFL view. With the medial border of the foot elevated to $15^{\circ}$ and the ankle in $45^{\circ}$ of plantar flexion (Fig. 1), the marker wire at the insertion of the ATFL appeared as an ellipse, because the central ray was tangential to the insertion (Fig. 3a). On this view the 'avulsed' fragment could be clearly seen (Fig. 4b), but the CFL fragment was superimposed on the lateral malleolus.

CFL view. Rotation medially by $45^{\circ}$ (Fig. 2) showed the CFL attachment without superimposition (Fig. 3b). In this CFL 


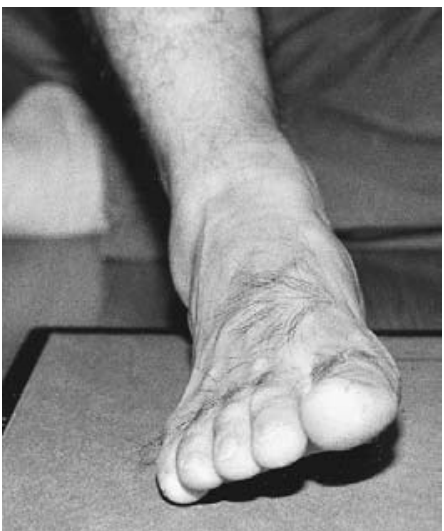

Fig. 1a

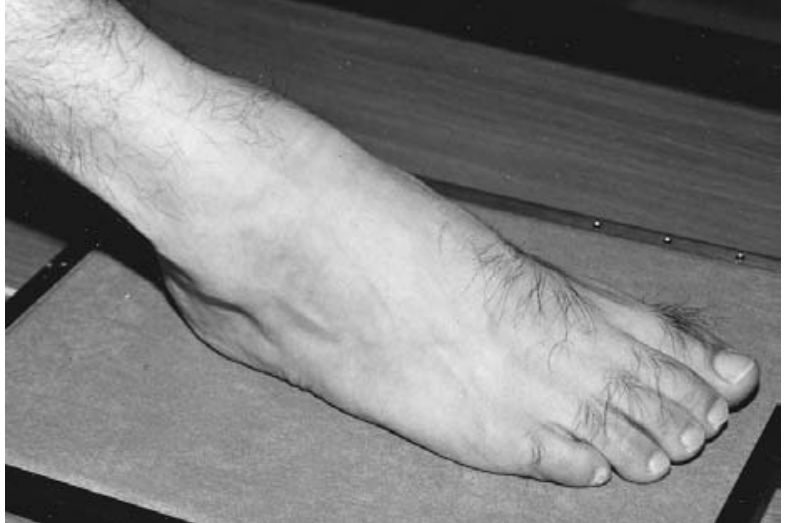

Fig. 1b

ATFL view. The medial border of the foot is elevated to $15^{\circ}$; the ankle is in $45^{\circ}$ of plantar flexion. The beam is directed vertically through the lateral malleolus to the cassette.

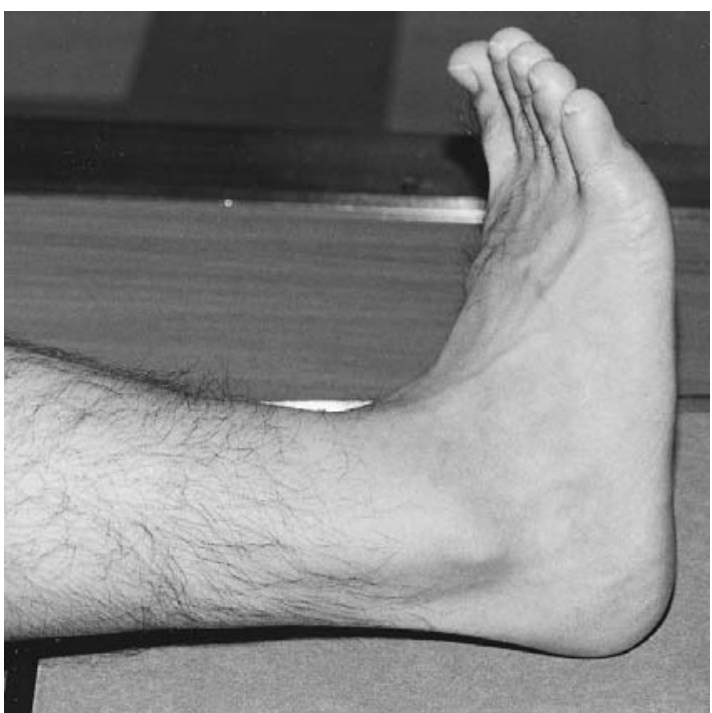

Fig. 2a

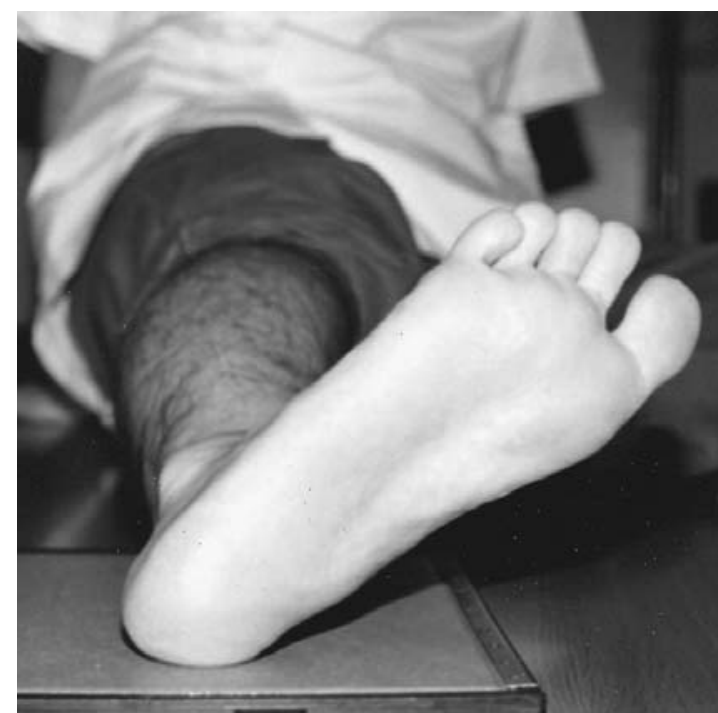

Fig. 2b

CFL view. The leg and foot are rotated $45^{\circ}$ medially from the position for the ankle mortise view with the foot in neutral position, that is 15 to $20^{\circ}$ more medial than for the traditional internal oblique projection. The beam is vertical through the lateral malleolus to the cassette.

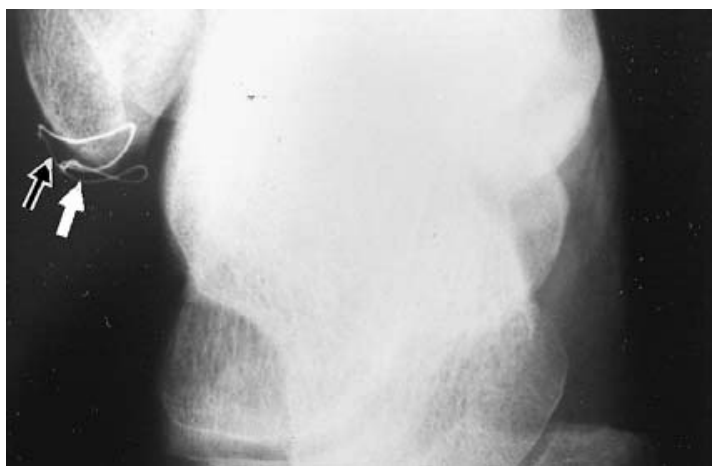

Fig. 3a

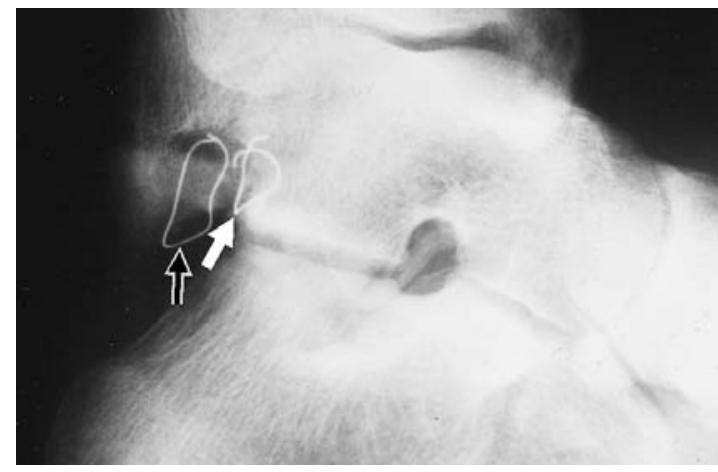

Fig. $3 b$

Views of the ankle with radiopaque markers around the insertions of the ligaments. The ATFL insertion (closed arrow) appears as an ellipse in that view (a). The CFL insertion (open arrow) is superimposed on the lateral malleolus. In the CFL view (b) the CFL insertion (open arrow) appears as the longest with no superimposition on other bony structures. The ATFL insertion (closed arrow) is superimposed on the talus. 


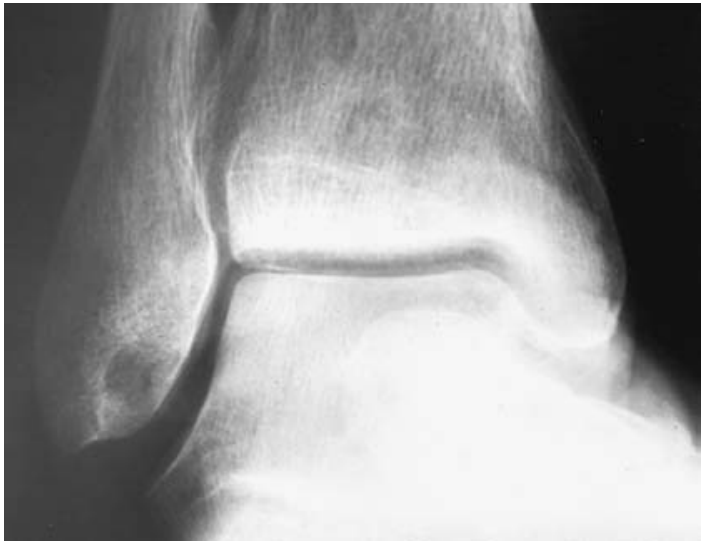

Fig. 4a

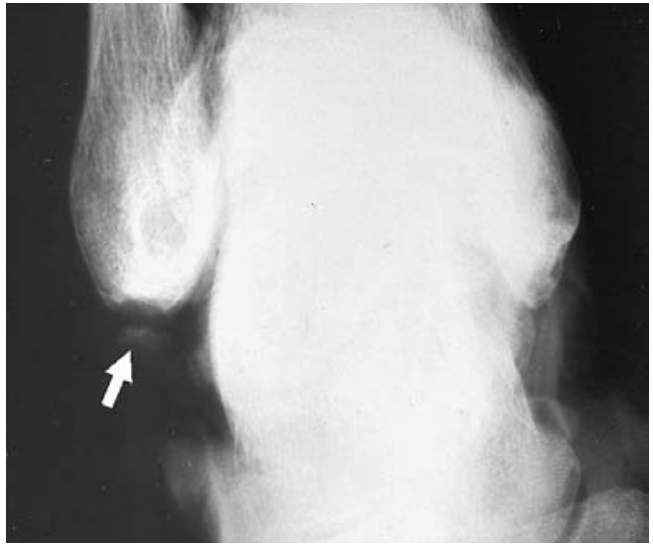

Fig. $4 b$

Radiographs of a simulated avulsion fracture of the ATFL. The ankle mortise view (a) shows that the fragment is superimposed on the lateral malleolus. In the ATFL view (b) the fragment (arrow) is clearly visible.

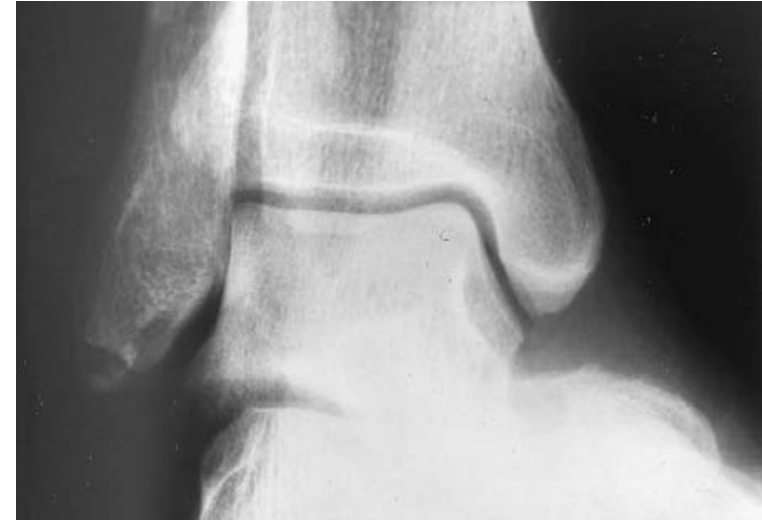

Fig. 5a

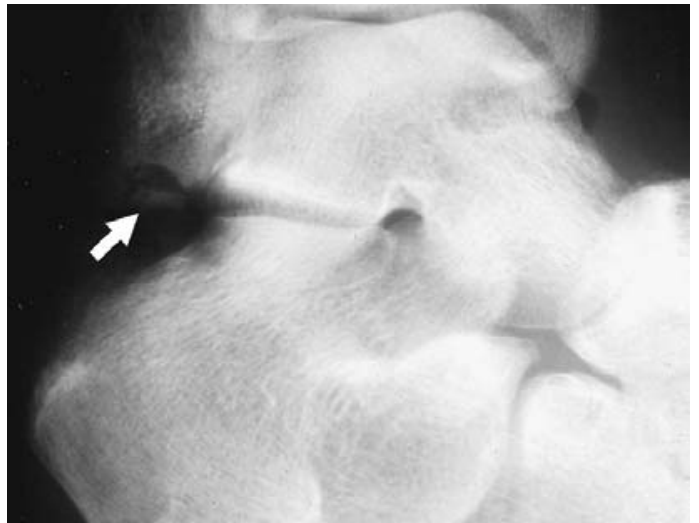

Fig. $5 b$

Radiographs of a simulated avulsion fracture of the CFL. The ankle mortise view (a) shows the fragment superimposed on the lateral malleolus. In the CFL view (b), the fragment (arrow) is clearly visible.

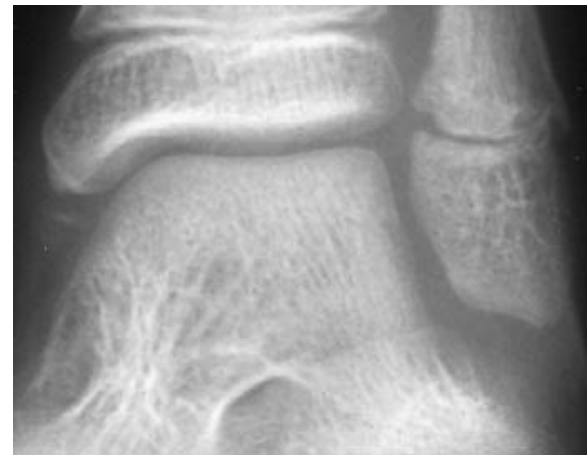

Fig. 6 a

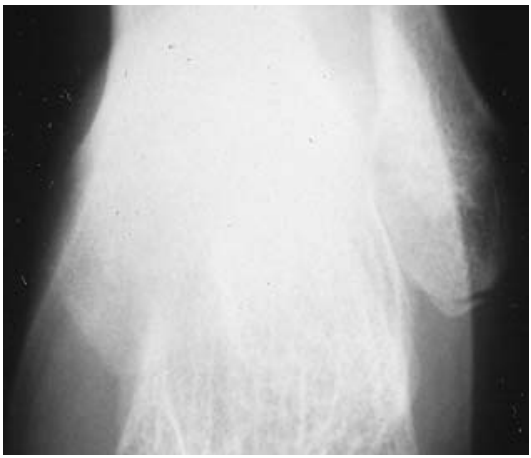

Fig. $6 \mathrm{~b}$

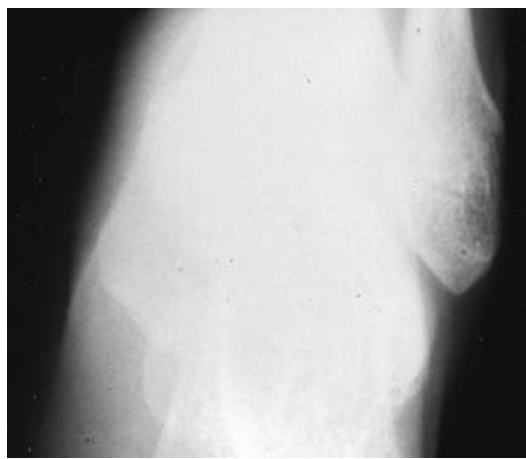

Fig. 6c

Radiographs of a 10-year-old boy after twisting his left ankle. The accessory centre of ossification in the medial malleolus was asymptomatic. An avulsion fragment could not be seen on the standard internal oblique view (a), but was clearly visible on the ATFL view (b). After six weeks in a cast, there is evidence of union on the ATFL view (c).

view, the ATFL fragment was superimposed on the talus.

On ankle mortise views, both the ATFL fragment (Fig. 4a) and the CFL (Fig. 5a) were superimposed on the lateral malleolus and could not be clearly seen.
Clinical application. Our radiographic projections can give accurate assessment of the displacement of the fragments without superimposition, and also clear radiographic distinction between ATFL and CFL avulsions (Figs 6 to 9). 


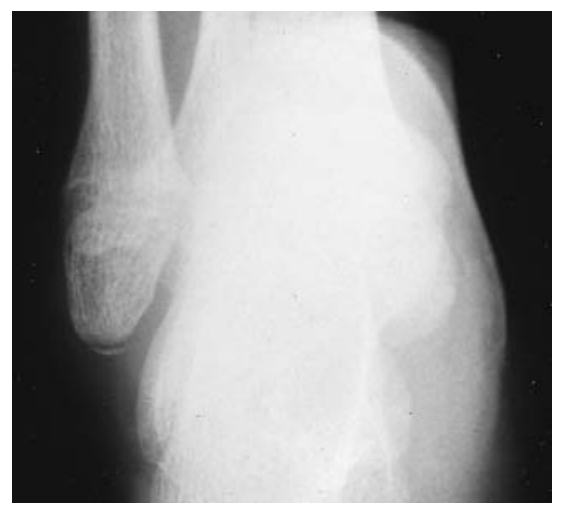

Fig. 7a

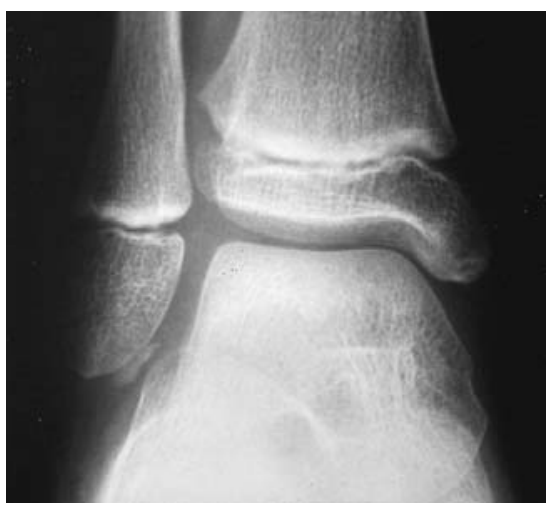

Fig. $7 b$

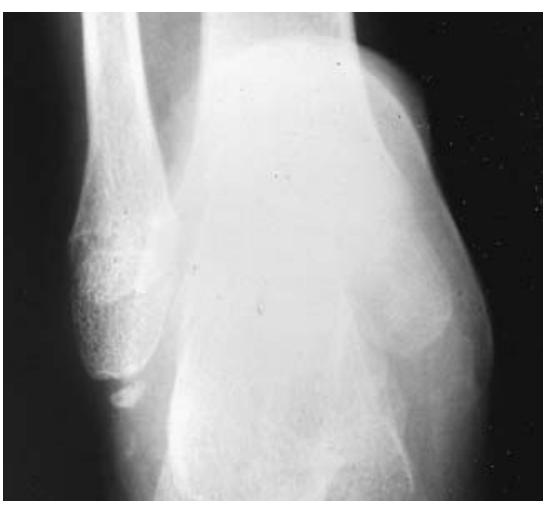

Fig. 7c

Radiographs of an eight-year-old girl after twisting her right ankle. The fragment was clearly visible on an the ATFL view (a). After use of a cast for seven weeks, the fracture failed to unite, and both mortise (b) and ATFL (c) views show the fragment at four months after injury.

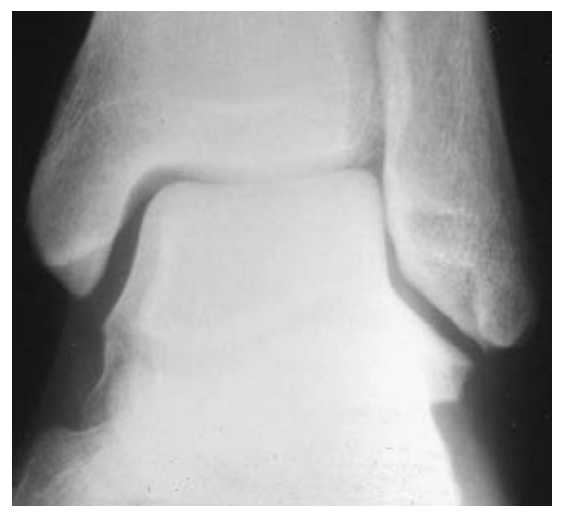

Fig. 8a

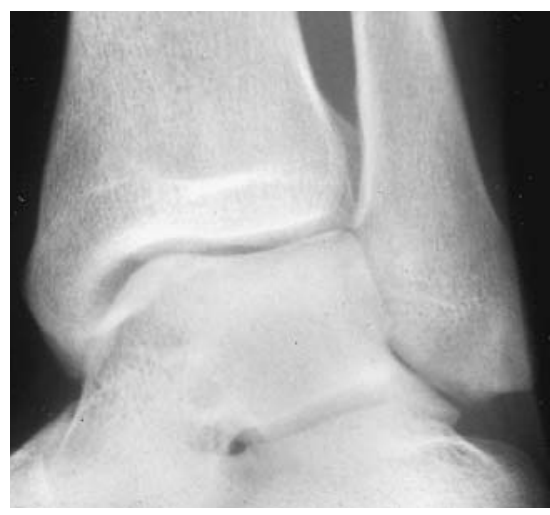

Fig. $8 \mathrm{~b}$

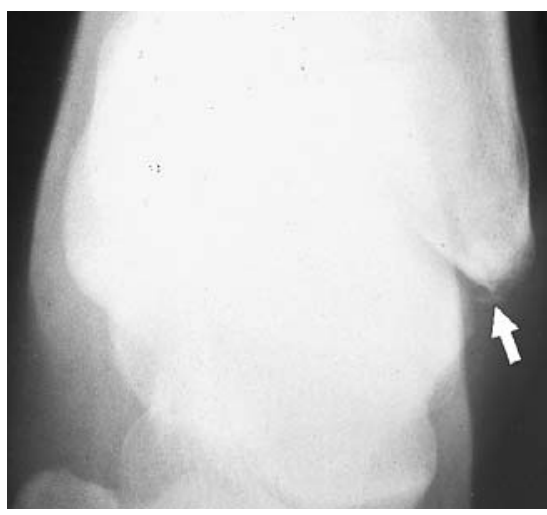

Fig. 8c

Radiographs of a 22-year-old man with a three-year history of recurrent instability of the left ankle. No fragment could be seen on the mortise (a) or the CFL (b) views, but one (arrow) was visible on the ATFL view (c). At operation, it was found to be attached to the proximal end of the ATFL.

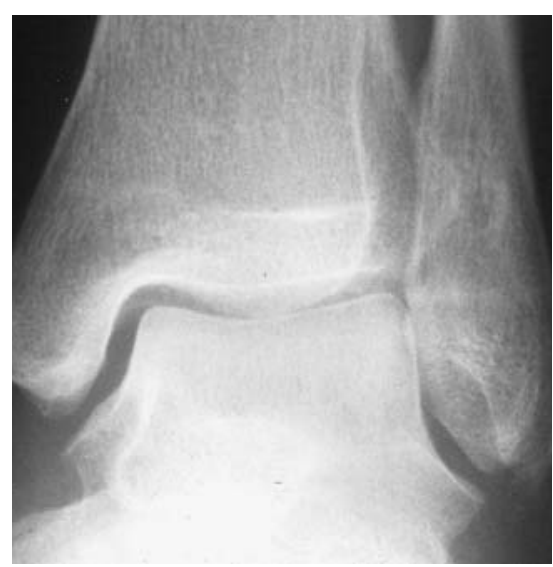

Fig. 9a

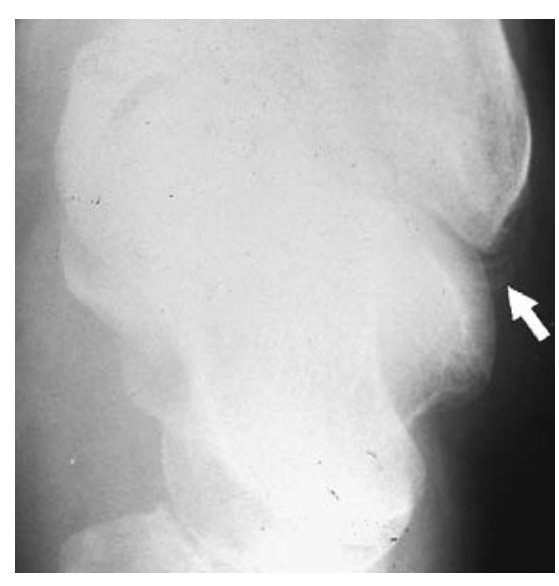

Fig. $9 \mathrm{~b}$

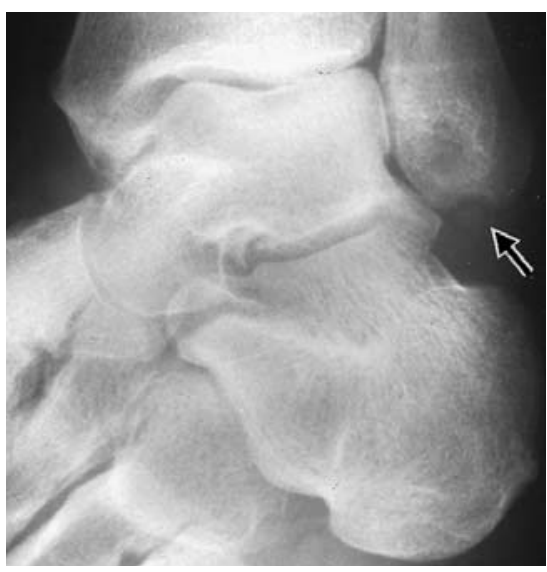

Fig. 9c

Radiographs of a 50-year-old woman with a five-year history of recurrent instability of the left ankle. On the mortise view (a), the fragments were superimposed and could not be clearly seen, but one fragment (closed arrow) was visible on the ATFL view (b) and another (open arrow) on the CFL view (c). At operation, both fragments were attached to the proximal end of the respective ligaments. 


\section{Discussion}

Broström $^{6}$ operated on 105 acute sprained ankles and found that 11 of 90 ATFL injuries had avulsed bony fragments, although three were not radiographically visible. Vahvanen, Westerlund and $\mathrm{Nikku}^{7}$ found cartilaginous or bony fragments in 19 of 40 acutely injured ankles in children, not visible radiographically in 11 . Busconi and Pappas ${ }^{3}$ confirm that in some skeletally immature patients radiographs often fail to show a bony fragment. The prevalence of bony avulsion may be higher than previously thought because avulsion fragments, particularly of the ATFL, are often barely visible on standard radiographs. Our projections will clearly show avulsion fragments (Figs 6 and 7).

The management of such avulsion fractures may be conservative or by surgery. The incidence of nonunion after conservative treatment is uncertain, because of the difficulties of estimating displacement and union on standard radiographs.

Chronic lateral pain or instability after an inversion sprain may be due to ligament damage, osteochondral fracture of the talus, subluxation of the peroneal tendon or anterolateral talar impingement. An ununited avulsion fracture at the lateral malleolus may be the cause even when standard radiographs fail to show it (Fig. 8). We now routinely use the new projections for patients with severe inversion injuries or complaints of chronic ankle instability.

No benefits in any form have been received or will be received from a commercial party related directly or indirectly to the subject of this article.

\section{References}

1. Berg EE. The symptomatic os subfibulare: avulsion fracture of the fibula associated with recurrent instability of the ankle. J Bone Joint Surg [Am] 1991;73-A:1251-4.

2. Broström L. Sprained ankles. VI. Surgical treatment of 'chronic' ligament ruptures. Acta Chir Scand 1966;132:551-65.

3. Busconi BD, Pappas AM. Chronic, painful ankle instability in skeletally immature athletes: ununited osteochondral fractures of the distal fibula. Am J Sports Med 1996;24:647-51.

4. Danielsson LG. Avulsion fracture of the lateral malleolus in children. Injury 1980;12:165-7.

5. Griffiths JD, Menelaus MB. Symptomatic ossicles of the lateral malleolus in children. J Bone Joint Surg [Br] 1987;69-B:317-9.

6. Broström L. Sprained ankles I: anatomic lesions in recent sprains. Acta Chir Scand 1964;128:483-95.

7. Vahvanen V, Westerlund M, Nikku R. Lateral ligament injury of the ankle in children: follow-up results of primary surgical treatment. Acta Orthop Scand 1984;55:21-5. 